\title{
Spin waves in terbium. III. Magnetic anisotropy at zero wave vector
}

\author{
Houmann, Jens Christian Gylden; Jensen, J.; Touborg, P.
}

\section{Published in:}

Physical Review B

Link to article, DOI:

10.1103/PhysRevB.12.332

Publication date:

1975

Document Version

Publisher's PDF, also known as Version of record

Link back to DTU Orbit

Citation (APA):

Houmann, J. C. G., Jensen, J., \& Touborg, P. (1975). Spin waves in terbium. III. Magnetic anisotropy at zero wave vector. Physical Review B, 12(1), 332-344. https://doi.org/10.1103/PhysRevB.12.332

\section{General rights}

Copyright and moral rights for the publications made accessible in the public portal are retained by the authors and/or other copyright owners and it is a condition of accessing publications that users recognise and abide by the legal requirements associated with these rights.

- Users may download and print one copy of any publication from the public portal for the purpose of private study or research.

- You may not further distribute the material or use it for any profit-making activity or commercial gain

- You may freely distribute the URL identifying the publication in the public portal

If you believe that this document breaches copyright please contact us providing details, and we will remove access to the work immediately and investigate your claim 


\title{
Spin waves in terbium. III. Magnetic anisotropy at zero wave vector
}

\author{
J. G. Houmann and J. Jensen \\ Danish Atomic Energy Commission Research Establishment Risø, Roskilde, Denmark \\ P. Touborg \\ Technical University, Lyngby, Denmark \\ (Received 6 May 1974)
}

\begin{abstract}
The energy gap at zero wave vector in the spin-wave dispersion relation of ferromagnetic $\mathrm{Tb}$ has been studied by inelastic neutron scattering. The energy was measured as a function of temperature and applied magnetic field, and the dynamic anisotropy parameters were deduced from the results. The axial anisotropy is found to depend sensitively on the orientation of the magnetic moments in the basal plane. This behavior is shown to be a convincing indication of considerable two-ion contributions to the magnetic anisotropy at zero wave vector. With the exception of the sixfold basal-plane anisotropy of the unstrained lattice, the dynamic anisotropy parameters deduced from our results agree with macroscopic measurements both with respect to the magnitudes (at zero temperature) and the temperature dependences. The deviations observed cannot be explained by existing theories which include the effects of zero-point deviations from the fully aligned ground state, and we tentatively propose polarization-dependent two-ion couplings as their origin.
\end{abstract}

\section{INTRODUCTION}

In this paper we shall study the behavior of the energy gap which is present at zero wave vector in the spin-wave spectrum of $\mathrm{Tb}$ as a consequence of magnetic anisotropy. We shall utilize some of the results obtained in the two preceding papers concerning the spin waves in $\mathrm{Tb}$, to be referred to as I and II (I: two-ion magnetic anisotropy; II: magnon-phonon interaction).

Because of the importance for the magnon energies of single-ion anisotropy in the long-wavelength limit, the general single-ion Hamiltonian for an hcp lattice is examined in detail. An explicit account of the magnetoelastic effects for a basal-plane ferromagnet is given, in which twoion magnetoelastic couplings are also included. We touch on the discussion of a "frozen"- versus a "flexible"-lattice model, and it is argued that the frozen-lattice model is correct when zeropoint deviations due to magnon-magnon and magnon-phonon interactions are neglected. Including anisotropic two-ion couplings in the spin Hamiltonian, we deduced a general expression for the energy gap of a basal-plane ferromagnet in I. Here we derive an expression for the energy gap in terms of static parameters which are determined by macroscopic experiments.

We have studied the spin-wave energy gap in $\mathrm{Tb}$ by inelastic neutron scattering. The energy gap as a function of magnetic field applied in the easy and hard directions in the basal plane was measured in a temperature range from 4.2 to $130 \mathrm{~K}$. The results were corrected for the field dependence of the relative magnetization at finite tem- peratures. The dynamic anisotropy parameters deduced by a least-squares fitting to the experimental results are comparable with the corresponding static parameters obtained from other measurements. Only the static and the dynamic values for the hexagonal anisotropy of the unstrained lattice fail to agree, which may be due to zero-point deviations. However, we suggest an alternative explanation. Finally, we discuss the possibility of large contributions to the magnetic anisotropy in the long-wavelength limit from anisotropic two-ion couplings and conclude that such contributions are very likely to occur.

\section{SINGLE-ION AND TWO-ION ANISOTROPY}

Starting from a general spin Hamiltonian of I an expression for the energy gap at zero wave vector in the spin-wave spectrum was deduced, Eqs. (21)-(25) of I, valid for a basal-plane ferromagnet. The different terms describing the properties of the energy gap in $\mathrm{Tb}$, which we obtained by studying the field dependence of the energy, may be classified according to the expressions derived in I. Because of the importance of the single-ion terms in the long-wavelength limit of the magnons, we shall here examine explicitly the single-ion Hamiltonian, bearing in mind that two-ion anisotropic couplings may influence the energy gap, as is apparent from Eqs. (22) and (23) of I.

The electrostatic field in which the ions are situated affects the energy levels of the system. Because the crystal-field energies are small compared to the spin-orbit coupling of the $4 f$ elec- 
trons of the rare-earth ions, the electrostatic energy of these electrons may be written

$$
\begin{gathered}
\mathcal{H}_{\mathrm{cf}}=\frac{1}{2} \sum_{i} \sum_{l m} \frac{1}{S_{l}}\left[V_{l m} \tilde{O}_{l, m}\left(J_{i}\right)+(-1)^{m} V_{l m}^{*} \tilde{O}_{l,-m}\left(J_{i}\right)\right], \\
m \geqslant 0,
\end{gathered}
$$

using the Wigner-Eckart theorem. $V_{l m}$ are the crystal-field parameters [corresponding to $K_{0 t 0}^{0 m 0}(0)$ in Eq. (7) of I]. The various other quantities occuring in (1) have been defined in $I$. Time-reversal symmetry requires $l$ to be even, and in the case of $f$ electrons only terms for which $l$ is smaller than or equal to 6 will be present. The symmetry of the electric field, which corresponds to the symmetry of the lattice, allows a reduction of (1). The structure of the heavy-rare-earth metals implies that $V_{l m}$ is real and $m$ is equal to 0 or 6 in these metals, which reduces the number of independent crystal-field parameters to four.

If the angular moments in the crystal are magnetically ordered, the total magnetoelastic energy of the system may be reduced by deformations of the lattice. Mason ${ }^{1}$ has derived a macroscopic expression for the magnetostriction, while Callen and Callen ${ }^{2}$ have developed a phenomenological theory based on group-theoretical representations for the allowed strains. Following the notation of Callen and Callen and of Cooper, ${ }^{3}$ we may write the homogeneous strains which transform according to the irreducible representations $\Gamma_{\alpha}, \Gamma_{\gamma}$, and $\Gamma_{\epsilon}$ of the point group of the hep lattice:

$$
\begin{aligned}
& \epsilon^{\alpha, 1}=\epsilon_{11}+\epsilon_{22}+\epsilon_{33}, \epsilon^{\alpha, 2}=\epsilon_{33}-\frac{1}{3} \epsilon^{\alpha, 1}, \\
& \epsilon^{\gamma}=\frac{1}{2}\left(\epsilon_{11}-\epsilon_{22}\right)+i \epsilon_{12}, \\
& \epsilon^{\epsilon}=\epsilon_{13}+i \epsilon_{23},
\end{aligned}
$$

where the strains $\epsilon_{i j}$ are defined as usual in terms of the elastic displacements $\overrightarrow{\mathrm{u}}(\overrightarrow{\mathrm{x}}, t)$,

$$
\epsilon_{i j}=\frac{1}{2}\left(\frac{\partial u_{i}}{\partial x_{j}}+\frac{\partial u_{j}}{\partial x_{i}}\right)
$$

where the Cartesian 1, 2, and 3 axes are along an $a, b$, and $c$ direction of the hcp lattice, respectively. The $\alpha$ strains are then symmetrypreserving dilatations along the $c$ axis and in the basal plane, the $\gamma$ strains are distortions of hexagonal symmetry of the basal plane, and the $\epsilon$ strains are $c$-axis shear modes. The elastic density associated with the homogeneous strains is

$$
\begin{aligned}
\mathcal{H}_{e}= & \frac{1}{2} c_{1}^{\alpha}\left(\epsilon^{\alpha, 1}\right)^{2}+c_{12}^{\alpha} \epsilon^{\alpha, 1} \epsilon^{\alpha, 2}+\frac{1}{2} c_{2}^{\alpha}\left(\epsilon^{\alpha, 2}\right)^{2} \\
& +\frac{1}{2} c^{\gamma} \epsilon^{\gamma}\left(\epsilon^{\gamma}\right)^{*}+\frac{1}{2} c^{\epsilon} \epsilon^{\epsilon}\left(\epsilon^{\epsilon}\right)^{*},
\end{aligned}
$$

where the five independent elastic constants can be expressed in terms of the Cartesian elastic constants $c_{i j}$, as in Cooper ${ }^{3}$ and Goodings and Southern. ${ }^{4}$ For the present purposes we shall define the reduced elastic constants

$$
\begin{aligned}
& c_{\gamma}=(1 / N J) c^{\gamma}=(1 / N J) 2\left(c_{11}-c_{22}\right), \\
& c_{\epsilon}=(1 / N J) c^{\epsilon}=(1 / N J) 4 c_{44},
\end{aligned}
$$

where $N$ is the number of ions per unit volume.

In the limit of small deformations, we can truncate a power expansion of the change of the crystal field at the first term linear in the strains. In this approximation, the modification of the crystal-field energy introduced by distortions of the lattice is exhaustively described by means of 17 independent single-ion parameters:

$$
\begin{aligned}
\mathcal{H}_{\mathrm{cf}-\mathrm{e}}=- & \frac{1}{2} \sum_{i} \sum_{l} \frac{1}{S_{l}}\left(\sum _ { m = 0 , 6 } \left(B_{l m}^{(1)} \epsilon^{\alpha, 1}\right.\right. \\
& \left.+B_{l m}^{(2)} \epsilon^{\alpha, 2}\right)\left[\tilde{O}_{l, m}\left(J_{i}\right)+\tilde{O}_{l,-m}\left(J_{i}\right)\right] \\
& +\sum_{m=-1,5} B_{l m}\left[\epsilon^{\epsilon} \tilde{O}_{l, m}\left(J_{i}\right)-\left(\epsilon^{\epsilon}\right) * \tilde{O}_{l,-m}\left(J_{i}\right)\right] \\
& \left.+\sum_{m=-2,4} B_{l m}\left[\epsilon^{\gamma} \tilde{O}_{l, m}\left(J_{i}\right)+\left(\epsilon^{\gamma}\right) * \tilde{O}_{l,-m}\left(J_{i}\right)\right]\right) .
\end{aligned}
$$

As in (1) we use a coordinate system with the $z$ axis along the $c$ direction, and $l$ is again restricted to the value 2,4 , and 6 . The effect on the energy gap of the magnetoelastic terms for which $l$ is equal to 2 was first considered by Turov and Sharov 5 and was later discussed at length by Cooper. ${ }^{3}$

The total magnetoelastic single-ion Hamiltonian is then a sum of the crystal field energy of the unstrained lattice, the elastic energy, and the energy of the coupling between the spin system and the lattice, $\mathcal{F}_{\mathrm{cf}-\mathrm{e}}$. In the presence of an external magnetic field, we must add the Zeeman energy $\mathfrak{H}_{Z}$ [Eq. (28) of I], and the single-ion Hamiltonian is then given by

$$
\mathfrak{H}_{\mathrm{J}}=\mathfrak{H C}_{\mathrm{cf}}+\mathcal{H}_{e}+\mathfrak{H}_{\mathrm{cf}-\mathrm{e}}+\mathcal{H}_{Z}
$$

If anisotropic two-ion interactions between the magnetic moments are neglected, the presence of magnetic anisotropy is related to the singleion anisotropy only. In most experiments and theoretical calculations on heavy-rare-earth metals, the two-ion coupling is assumed to be an isotropic Heisenberg interaction, [Eq. (1) of I]. We shall start with this assumption and consider later the possible effects of two-ion anisotropy.

In equilibrium the derivatives of the free energy with respect to the strains must be zero, e.g., 
$\left\langle\frac{\partial \mathcal{F}_{J}}{\left(\partial \epsilon^{\gamma}\right)^{*}}\right\rangle=c^{\gamma} \bar{\epsilon}^{\gamma}-\sum_{l, m=-2,4} \frac{1}{S_{l}} B_{l m}\left\langle\sum_{i} \tilde{O}_{l,-m}\left(J_{i}\right)\right\rangle=0$.

The $\gamma$ and $\epsilon$ strains are zero unless the moments are ferromagnetically ordered, or, put in another way, the equilibrium strains are always homogeneous throughout the crystal. This is called the "lattice-clamping" effect and has been treated in detail by Evenson and Liu. ${ }^{6}$ The $\gamma$ and $\epsilon$ contributions to the free energy are then zero in a conical or helically ordered phase. The lowering of the magnetoelastic energy in passing from the helical to ferromagnetic state is the basic driving force behind the ferromagnetic transition in ${ }^{7}$ Dy and probably also in $\mathrm{Tb}$, as proposed by Cooper ${ }^{3,8}$

In the ferromagnetic phase of $\mathrm{Tb}$, where the moments lie in the basal plane, the $\gamma$ strains are related to the magnetostriction coefficients defined by Mason ${ }^{1}$ in the following way:

$$
\bar{\epsilon}^{\gamma}=C e^{2 i \phi}-\frac{1}{2} A e^{-4 i \phi},
$$

where $\phi$ is the angle between the direction of magnetization and the 1 axis. Combining (8) and (9), we obtain at zero temperature

$$
\begin{aligned}
& C=\frac{1}{J c_{\gamma}} \sum_{l} B_{l 2} \Gamma_{l, 2}, \\
& A=-2 \frac{1}{J c_{\gamma}} \sum_{l} B_{l 4} \Gamma_{l, 4},
\end{aligned}
$$

using the expectation values deduced in I for the Racah operators $[(11)-(13)]$. The magnetostric tion coefficients, which have been determined experimentally for $\mathrm{Tb}$ by Rhyne and Legvold, ${ }^{9}$ are proportional to certain linear combinations of the coupling parameters defined in (6).

The $\epsilon$-strain part of (6) vanishes for a basalplane ferromagnet, which follows from the fact that $l+m$ is odd in this case. If $B_{66}^{(i)}$ in the $\alpha-$ strain part of (6) is neglected, this part may be taken into account by introducing the effective crystal-field parameters:

$$
V_{l 0}^{\prime}=V_{l 0}-\left(\boldsymbol{B}_{l 0}^{(1)} \bar{\epsilon}^{\alpha, 1}+B_{l 0}^{(2)} \bar{\epsilon}^{\alpha, 2}\right) .
$$

Later we shall see that this approximation is acceptable in the case of $\mathrm{Tb}$.

When the equilibrium strains are introduced in (7), the reduced magnetoelastic energy (in the case $\theta=\frac{1}{2} \pi$ and $T=0 \mathrm{~K}$ ) is

$$
\begin{aligned}
E_{J}=\frac{1}{N J}\left\langle\mathcal{H}_{J}\right\rangle= & \frac{1}{J} \sum_{l} \Gamma_{l, 0} V_{l 0}^{\prime}-\frac{1}{2} c_{\gamma} C^{2}-\frac{1}{8} c_{\gamma} A^{2} \\
& +\left(\frac{1}{J} \Gamma_{6,6} V_{66}+\frac{1}{2} c_{\gamma} A C\right) \\
& \times \cos 6 \phi-g \mu_{B} H \cos (\delta-\phi),
\end{aligned}
$$

where $\delta$ is the angle between the direction of the applied field and the 1 axis. The sign of the coefficient of $\cos 6 \phi$ determines the easy planar axis. This coefficient is positive in the case of $\mathrm{Tb}$, which corresponds to an alignment of the moments parallel with a $b$ axis $(\phi=\pi / 6+p \pi / 3)$ in the absence of an external field. If a field is applied along a hard planar axis $(\delta=0)$ then the magnetization is pulled towards this direction, as described by the equation

$g \mu_{B} H=g \mu_{B} H_{c}(\sin 6 \phi) / 6 \sin \phi$

$$
=g \mu_{B} H_{c} \frac{1}{3}\left(3-16 \sin ^{2} \phi+16 \sin ^{4} \phi\right) \cos \phi,
$$

as long as the field is smaller than the critical field $H_{c}$,

$$
g \mu_{B} H_{c}=36(1 / J) \Gamma_{6,6} V_{66}+18 c_{\gamma} A C .
$$

At this field the moments are pulled fully around to the hard axis $(\phi=0)$.

Because of the presence of magnetic anisotropy, the spin-wave dispersion relation in $\mathrm{Tb}$ exhibits an energy gap, $\Delta$, at zero wave vector. This energy gap may easily be calculated by using the expansion in spin deviation operators of the Racah operators introduced in I [Eqs. (16) and (17)]:

$$
\begin{aligned}
\Delta=\boldsymbol{\varepsilon}(0)= & \left\{\left[\boldsymbol{A}(0)+\boldsymbol{B}(0)+g \mu_{B} H \cos (\delta-\phi)\right]\right. \\
& \left.\times\left[A(0)-B(0)+g \mu_{B} H \cos (\delta-\phi)\right]\right\}^{1 / 2},
\end{aligned}
$$

where we shall define the following quantities:

$$
A(0) \pm B(0)=P_{0}( \pm)-P_{6}( \pm) \cos 6 \phi .
$$

We then have

$$
\begin{aligned}
P_{0}(+)= & -\frac{1}{J} \sum_{l} l(l+1) \Gamma_{l, 0} V_{l 0}^{\prime}+14 C \frac{1}{J} \Gamma_{4,2} B_{42} \\
& +36 C \frac{1}{J} \Gamma_{6,2} B_{62}-11 A \frac{1}{J} \Gamma_{6,4} B_{64} \\
& +2 c_{\gamma} C^{2}+c_{\gamma} A^{2}+N_{c} g \mu_{B} M \\
P_{6}(+)= & 6(1 / J) \Gamma_{6,6} V_{66}+3 c_{\gamma} A C+\Delta M \\
P_{0}(-)= & 4 c_{\gamma}\left(C^{2}+A^{2}\right)+N_{\perp} g \mu_{B} M \\
P_{6}(-)= & 10 c_{\gamma} A C+36(1 / J) \Gamma_{6,6} V_{66}
\end{aligned}
$$

The parameter $\Delta M$ in $(17 \mathrm{~b})$ which is defined as the difference between $P_{6}(+)$ and $\frac{1}{6} g \mu_{B} H_{c}$ was introduced by Lindgard ${ }^{10}$ and in the present approximations is given by

$$
\begin{aligned}
\Delta M & =P_{6}(+)-\frac{1}{6} g \mu_{B} H_{C} \\
& =7 A \frac{1}{J} \Gamma_{4,2} B_{42}+18 A \frac{1}{J} \Gamma_{6,2} B_{62}-22 C \frac{1}{J} \Gamma_{6,4} B_{64} .
\end{aligned}
$$

In (17) we have included the effect of the demagnetization field as deduced by Keffer. ${ }^{11} N_{c}$ and 
$N_{\perp}$ are the demagnetization factors, respectively, in the $c$ direction and in the basal plane perpendicular to the magnetization $\vec{M}$. The field which enters into (13) and (15) is then the internal field. $B_{66}^{(i)}$, which has been neglected, would have given rise to terms proportional to $\cos ^{2} 6 \phi$ in both $A(0)$ $+B(0)$ and $A(0)-B(0)$.

If $C$ and $A$ are both zero, then $P_{0}(-)=0$ (except for the demagnetization contribution) and $P_{6}(-)$ $=g \mu_{B} H_{c}$, and in this case the second-order transition at $H=H_{c}$ ( $H$ parallel to a hard axis) is accompanied by a vanishing of the energy gap $\Delta$. The $\gamma$-strain contributions, which prevent the uniform magnon mode from going soft, were first considered by Turov and Sharov, ${ }^{5}$ and, because only the static parts of the strains have been introduced, they called it the "frozen-lattice" approximation. The time dependence of the inhomogeneous parts of the strains in (6) may be expanded in phonon operators and, as such, give rise to magnon-phonon interaction. ${ }^{12}$ The homogeneous strains are essentially classical quantities, which result from the vanishing of the natural vibration energy of the lattice. On this basis Jensen, ${ }^{13}$ Chow and Keffer, ${ }^{14}$ and $\mathrm{Liu}^{15}$ have shown that the presence of the phonons in the systems has negligible effect on the magnon energy gap. In an attempt to explain the observation of ferromagnetic resonances in Dy and $\mathrm{Tb}^{16}$ at much lower frequencies than expected, Cooper ${ }^{3}$ proposed a flexible- or free-lattice model, which was based on the free energy of the system. In this model and in the equivalent one suggested by Vigren and $\mathrm{Liu}^{17}$ the energy gap vanishes at $H=H_{c}$ even in the case where the $\gamma$ strains are nonzero. The previous assumptions for the dynamics of the spinlattice system have been demonstrated to be incorrect by the neutron-scattering experiment performed on $\mathrm{Tb}-10 \%$-at.-Ho, ${ }^{18}$ and by the solution of the equations of motion ${ }^{13-15}$ (see also II). Although (when the phonons are introduced) the lattice is completely "free," the spins will perceive the lattice as being "frozen." This apparent paradox is explained by the finite mass of the ions or, completely equivalently, by the vanishing of the eigenfrequency of the lattice at zero wave vector; the lattice cannot respond to a uniform spin precession at finite frequency.

As discussed, the uniform mode does not go soft at the transition at $H=H_{c}$ if $\gamma$-strain contributions are present. Instead, it is predicted $d^{13-15}$ that the long-wavelength phonons corresponding to the $\gamma$ strain go soft, in the sense that the sound velocity $\left(\omega_{q} / q\right)$ goes to zero. The phonons in question are the acoustic transverse phonons with propagation and polarization vectors both lying in the basal plane (parallel or perpendicular to the magnetization). The soft-phonon behavior has been observed experimentally ${ }^{19,20}$ at the JahnTeller phase transition in various rare-earth salts which represent systems equivalent to the one considered. Preliminary ultrasonic measurements on $\mathrm{Tb}$ indicate a softening of the phonons at $H=H_{c}{ }^{21}$ These measurements are continuing, and one of the purposes is to clarify further the behavior of the basal-plane anisotropy in $\mathrm{Tb}$ determined by the present work. The soft-mode behavior in the metals may be affected by the existence of two-ion exchange interactions.

Referring to the equilibrium conditions for the strains, Eq. (8), it appears that the terms in the general spin Hamiltonian (7) in I, for which $\lambda$ is different from zero, account for the magnetoelastic contributions. However, as described in I [mechanism (v)], such terms may occur also because of the polarization and the spin-orbit coupling of the conduction electrons, which modify the susceptibility and thus the indirect exchange interaction. Depending on the signs of the $\lambda$ terms which are not of magnetoelastic origin, they may either enhance or resist the softening of the phonons. From the experimental results in $\mathrm{Tb}$ we isolate a contribution to the basal-plane anisotropy which is tentatively explained by this mechanism (v). The sign of this term is such as to enhance the softening of the transverse-phonon mode. The sound velocity may then vanish at a field smaller than the critical field, suggesting a structural change of the crystal at this field. See Note added in proof.

The introduction of two-ion interactions, as considered in I, still allows the energy gap to be written in the form given by (15) and (16), if terms in Eqs. (22) and (23) of I for which $\mu+m+m^{\prime}$ is greater than 6 are neglected. Twoion couplings for which $\lambda=0$ may contribute to all the energy-gap parameters, $P_{i}( \pm)$, with the exception of $P_{0}(-)$, for which $m+m^{\prime}$ in Eq. (23) of $I$ is equal to zero. An explicit calculation of possible magnetoelastic contributions $(\lambda \neq 0)$ to the energy gap shows that the $\gamma$-strain terms appearing in $g \mu_{B} H_{c}, P_{0}(-)$, and $P_{6}(-)$ can still be written in terms of $C$ and $A$ as given by (14), (17c), and (17d), where $C$ and $A$ defined by (9) include the single-ion contributions (10) as well as twoion contributions. In this context we mention that "optical" strain contributions can be neglected because of the vanishing of the electric dipole moment of the ions [see the mechanisms (vi) and (vii) in I] and of the acoustic-optical coupling at $q=0$, as discussed in $\Pi$. Then within a linear theory the strains of the two hcp sublattices will have the same signs, and only "acoustic" strains are present. 
In general it is not possible to distinguish between single-ion and two-ion contributions to the energy gap or to the static magnetic anisotropy. Only the strain-independent two-ion terms discussed above, for which $\lambda$ is nonzero, may show up as differences between the energy-gap parameters and the corresponding ones obtained from macroscopic measurements. Torque or magnetization measurements are in most cases interpreted in terms of the single-ion crystal-field parameters of the undistorted lattice, Eq. (1). The magnetic anisotropy energy is assumed to depend on $\theta$ and $\phi$ in the following way ${ }^{22}$ :

$$
\begin{aligned}
F_{A}(\theta, \phi)= & K_{2} P_{2}^{0}(\cos \theta)+K_{4} P_{4}^{0}(\cos \theta)+K_{6} P_{6}^{0}(\cos \theta) \\
& +K_{6}^{6} \sin ^{6} \theta \cos 6 \phi
\end{aligned}
$$

where $P_{l}^{m}(\cos \theta)$ are the Legendre polynomials. Because of the strong axial anisotropy in $\mathrm{Tb}$ (and Dy) it is possible to compare the effective parameters defined by Eq. (19) with those derived from a more general (and realistic) Hamiltonian including terms whose angular dependences differ from those introduced in (19). In a torque or magnetization experiment on $\mathrm{Tb}$ or Dy the magnetic moments are pulled only a few degrees out of the basal plane towards the $c$ axis, and (19) can be approximated by the expansion

$$
\begin{aligned}
F_{A}(\theta, \phi)= & F_{A}\left(\frac{\pi}{2}, \phi\right)+\frac{1}{2 !} F_{A}^{(2)}\left(\frac{\pi}{2}, \phi\right) \delta \theta^{2} \\
& +\frac{1}{4 !} F_{A}^{(4)}\left(\frac{\pi}{2}, \phi\right) \delta \theta^{4}
\end{aligned}
$$

where $\delta \theta=\theta-\frac{1}{2} \pi$ and

$$
\begin{aligned}
F_{A}^{(2)}\left(\frac{\pi}{2}, \phi\right) & =\left(\frac{\partial^{2} F_{A}(\theta, \phi)}{\partial \theta^{2}}\right)_{\theta=\pi / 2} \\
& =3 K_{2}-\frac{15}{2} K_{4}+\frac{105}{8} K_{6}-6 K_{6}^{6} \cos 6 \phi .
\end{aligned}
$$

The true anisotropy energy may be written as a similar power expansion in $\delta \theta$, and the effective axial anisotropy parameters determined by experiments, $K_{l}^{m}$, may be considered as a measure of the second (and fourth) derivative of the free energy as given by $(21) . \quad F_{A}^{(2)}\left(\frac{1}{2} \pi, \phi\right)$ will represent the second derivative of the actual anisotropy also in cases where $K_{6}$ or $K_{4}$ and $K_{6}$ have been neglected in the interpretation of the anisotropy measurements.

As considered in $\mathrm{I}$, the spin-wave energy gap is closely related to the second derivatives of the free energy ${ }^{3}$ [Eqs. (24) and (25) of I]. If we neglect the terms in the general Hamiltonian (7) of I proportional to $\cos 12 \phi$ and $\cos 18 \phi(p \neq 0,1)$ and also the terms for which $\lambda$ is different from zero but which do not contribute to the elastic energy, we obtain the following expressions for the energy-gap parameters in terms of macroscopic parameters:

$$
\begin{aligned}
A(0)+B(0)= & (1 / N J) F_{A}^{(2)}\left(\frac{1}{2} \pi, \phi\right)+\frac{1}{4} c_{\epsilon} H_{0}^{2}+N_{c} g \mu_{B} M, \\
A(0)-B(0)= & 4 c_{\gamma}\left(C^{2}+A^{2}\right)-\left[36(1 / N J) K_{6}^{6}-8 c_{\gamma} A C\right] \cos 6 \phi \\
& +N_{\perp} g \mu_{B} M,
\end{aligned}
$$

where the basal-plane anisotropy is simply related to the critical field

$$
K_{6}^{6}=\frac{1}{36} N J g \mu_{B} H_{c} .
$$

In distinction to the $\alpha$ and $\gamma$ strains, the first derivatives of the $\epsilon$ strains with respect to $\theta$ are different from zero at $\theta=\frac{1}{2} \pi$, which is the reason for the presence of $\epsilon$-strain contributions to $(22 \mathrm{a})$. $H_{0}$ is considered to be an effective magnetostriction coefficient given by

$$
H_{0}=\frac{2}{J c_{\epsilon}} \sum_{l}[l(l+2)]^{1 / 2} \Gamma_{l+1,1} B_{l 1}
$$

when two-ion contributions and $B_{65}$ in (6) are neglected. The macroscopic expression for the energy gap, (22), is quite general because of the inclusion of two-ion interactions. The terms which give rise to $12 \phi$ and $18 \phi$ dependences are presumably small and are neglected. They would have modified the equilibrium equation determining $\phi,(13)$, and hence the magnitude of the critical field, and the transition at this field could be of first order.

The two-ion $\lambda$ terms, which are not of magnetoelastic origin, are not necessarily negligible and may lead to differences between the macroscopic energy-gap parameters, as given by (22), and the actual (microscopic) energy-gap parameters. Defining

$$
\delta_{i}( \pm)=\left[P_{i}( \pm)\right]_{\text {micr. }}-\left[P_{i}( \pm)\right]_{\text {macr. }}, \quad i=0,6,
$$

we have, e.g.,

$$
\begin{gathered}
\delta_{6}(-)=\sum_{\lambda \mu} \sum_{l m} \sum_{l^{\prime} m^{\prime}} \frac{1}{J} K_{\lambda l l^{\prime}}^{\mu m m^{\prime}(0) \mu(12-\mu)} \\
\times \Gamma_{\lambda, \mu} \Gamma_{l, m} \Gamma_{l^{\prime}, m^{\prime}},
\end{gathered}
$$

using the notation introduced in $\mathrm{I}\left(\mu+m+m^{\prime}=6\right)$. The corresponding expressions for the other differences may easily be obtained from the energygap expressions deduced in $\mathrm{I}[(21)-(25)]$. As quoted in $I$ [mechanism (iv)] the indirect exchange interaction perturbs the energy gap so to give rise to a relative shift of the order of $2.5 \%$, which will also appear in the differences $\delta_{i}( \pm)$. At low temperatures we may have additional contributions 
to $\delta_{i}( \pm)$ from the zero-point motion of the magnetic moments and of the ions. The zero-point effects due to magnon-magnon interactions can give rise to a deviation between static and dynamic measurements. The importance of these contributions increases with the strength of the anisotropy and with the rank $l$ of the tensor spin operators considered. In the Hartree-Fock calculation by Lindgård and Danielsen, ${ }^{23}$ in which twoion anisotropy has been neglected, the contributions to $\delta_{i}( \pm)$ in $\mathrm{Tb}$ arising from the magnon-magnon interactions are found to be quite small, except for the contributions to $\delta_{6}(-)$. The results by Lindgård and Danielsen do not seem to confirm the proposal by $\mathrm{Egami}^{24}$ of a strong $\phi$ dependence of the basal-plane anisotropy in Dy. The two-magnon-one-phonon scattering processes $^{13}$ may also give rise to zero-point contributions (see II); however, they are probably not as important as, and may hardly be distinguished from, those due to magnon-magnon interactions.

The equations above, which describe the behavior of the spin system at zero temperature, may be generalized to the case of finite temperature by the procedure outlined in $I,(31)-(35)$. The deviation of the relative magnetization $\sigma$ [Eq. (31) of I] from unity may be taken into account by replacing $J$ with $\sigma J$ in all formulas. In Eqs. (17) and (22) the magnetization $M$ is replaced by $M(0) \sigma^{2} / \sigma$ $=M(T)$. The strong anisotropy of the basal-plane ferromagnets $\mathrm{Tb}$ and $\mathrm{Dy}$ may in addition to a modification of the Callen and Callen theor $\mathrm{y}^{2}[\mathrm{Eq}$. (33) of I], introduce deviations from the simple spin-wave renormalization theory of Cooper, ${ }^{3}$ which should be of an appreciable magnitude in the case of $P_{6}(-)$ according to the results obtained by Lindgård and Danielsen. ${ }^{23}$

\section{ENERGY-GAP MEASUREMENTS}

The spin-wave energy gap in $\mathrm{Tb}$ at zero wave vector was studied by inelastic neutron scattering. Because of the finite energy of the long-wavelength magnons ( $1-2 \mathrm{meV})$, the Bragg reflection does not disturb the observation of the neutrons scattered by the magnons at zero wave vector. In Fig. 1 is shown the neutron groups obtained at $q=0$ (the scattering vector equals a reciprocallattice vector) in zero magnetic field and at maximum field applied in the hard direction of $\mathrm{Tb}$ at $40 \mathrm{~K}$. The sample was a monocrystalline disc with a diameter of about $14 \mathrm{~mm}$ and a thickness of about $5 \mathrm{~mm}$ and with the $c$ axis normal to the faces. From the saturation magnetization of $\mathrm{Tb},{ }^{25}$ the demagnetization field normal to the $c$ axis in the sample is deduced to be $N_{\perp} M(T)=\sigma \times 6.65 \mathrm{kOe}$. The energy gap as a function of magnetic field ap-
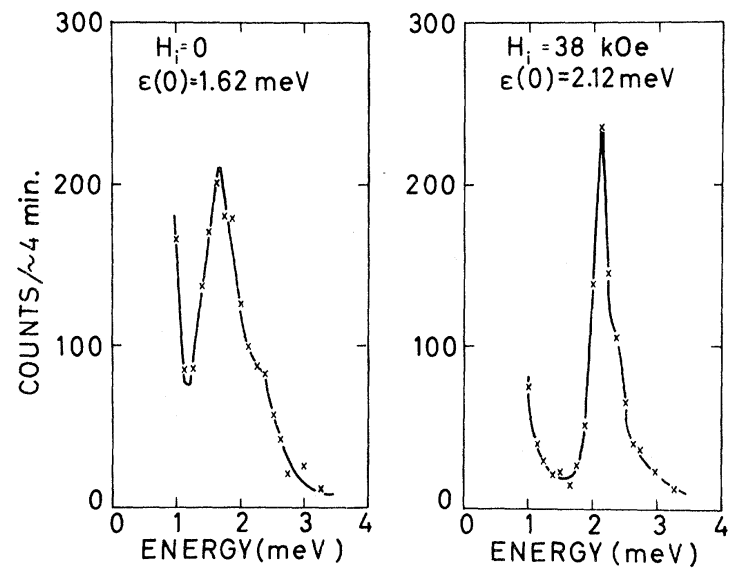

FIG. 1. Example of neutron groups obtained in $\mathrm{Tb}$ at zero wave vector. The scattering vector is along the $c$ axis and equal to $4 \pi / c$. The groups are measured at (a) $40 \mathrm{~K}$ in zero field and (b) an internal field equal to $38 \mathrm{kOe}$ applied in a hard direction.

plied along the easy and hard directions in the basal plane. was measured in a temperature interval ranging from 4.2 to $130 \mathrm{~K}$. In this temperature range, which corresponds to a relative magnetization between 1 and 0.8 , the field dependence of the magnetization is quite well described by the molecular-field result [Eq. (34) of I] as indicated by the magnetization measurements. ${ }^{25}$ Further, we have calculated the forced magnetostriction $d(2 C+A) / d H$ based on the magnetostriction theory of Callen and Callen ${ }^{2}$ by using the value obtained from Eq. (34) of I: $d \sigma / d H=(1-\sigma) 0.0027 \mathrm{kOe}^{-1}$, and found a fair agreement with the experimental results of Rhyne and Legvold ${ }^{9}$ for $\sigma>0.8$ (Fig. 2).

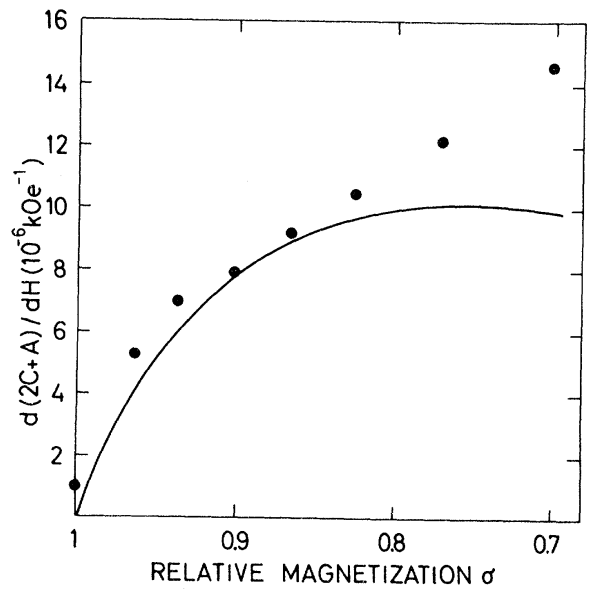

FIG. 2. Forced magnetostriction $d(2 C+A) / d H$ in $\mathrm{Tb}$ determined by experiments (Ref. 9) and calculated by using the magnetostriction theory of Callen and Callen (Ref. 2). The deviation at high temperatures $(\sigma<0.8)$ may be due to contributions which are not linear in field. 
The experimental procedure and the treatment of the results follow the lines discussed in $I$. The interpretation is facilitated by the vanishing of the direct coupling of magnons and phonons at zero wave vector (II). The effective $g$ value introduced in $\mathrm{I},(30)$, has not been used in the present treatment, partly because of the smallness of the correction and partly because it is expected to be cancelled by opposite contributions at $q=0$ arising from mechanism (iv) in I.

A selection of the experimental results for the energy gap at $q=0$ is shown in Fig. 3. They are qualitatively similar to earlier measurements ${ }^{18}$ on $\mathrm{Tb}-10 \%$-at.-Ho, but they are made with considerably greater precision, and in this experiment the effect of the field dependence of $\sigma$ is taken into account. This correction introduces changes of the magnetization exponents connected with the $A(0) \pm B(0)$ parameters, which are of the order of \pm 1 . In the $\mathrm{Tb}-10 \%$-at.-Ho experiment the number of independent parameters was reduced by adopting a particular model corresponding to Eqs. (14)-(18) if $\Delta M$ is neglected; an attempt was made to isolate the magnetoelastic contribution to the critical field, Eq. (14), by neglecting the possibility of a difference $\delta_{6}(-)$ between the macroscopic and microscopic parameters. The greater precision of the present experimental results for pure $\mathrm{Tb}$ allow us to avoid such assumptions. The only assumption made is that terms proportional to $\cos 12 \phi$ and $\cos 18 \phi$ can be neglected. The parameters which are deduced from the energy-gap measurements are then the four $P_{i}( \pm)$ defined by Eqs. (15) and (16)

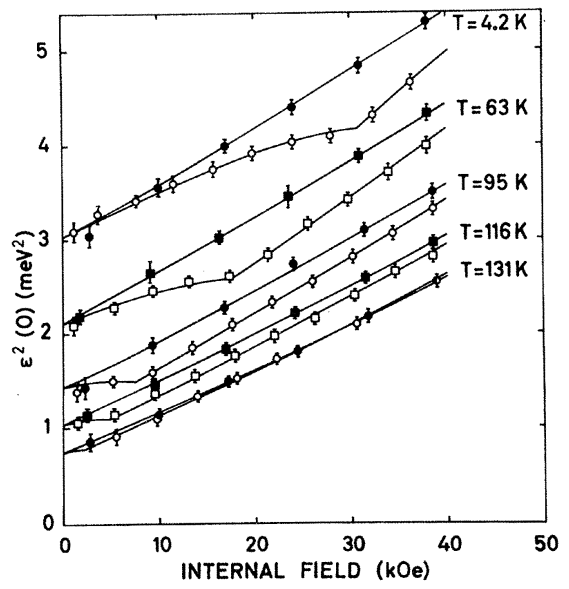

FIG. 3. Dependence of the square of the magonon energy gap on internal magnetic field in $\mathrm{Tb}$. Open symbols represent results for the field in the hard direction, and closed the easy direction. The full lines are leastsquares fits of the energy-gap expression, given in the text, to the experimental results. and the critical field, $H_{c}$. The abrupt changes of the field dependences of $\Delta^{2}$ when the field is applied in a hard direction (Fig. 3) serve as a welldefined determination of the critical field as a function of temperature. The field dependence of $\phi$ is then obtained from Eq. (13). The $\sigma$ dependence of $P_{i}( \pm)$ defined by

$$
P_{i}( \pm) \propto \sigma^{\gamma_{i}( \pm)}
$$

implies corrections to the energy-gap expression, (15) and (16), as given by

$$
\begin{aligned}
A(0) \pm B(0)= & P_{0}( \pm)-P_{6}( \pm) \cos 6 \phi \\
& +\frac{1}{\sigma}\left[\gamma_{0}( \pm) P_{0}( \pm)-\gamma_{6}( \pm) P_{6}( \pm) \cos 6 \phi\right] \\
& \times \frac{d \sigma}{d H}\left(H-H_{a}\right)
\end{aligned}
$$

where $H_{a}=0$ when the field is applied in an easy direction, and when applying the field along a hard axis $H_{a}=H$ for $H<H_{c}$ and $H_{a}=H_{c}$ for $H>H_{c}$ $\left(d \sigma / d H\right.$ is assumed to be zero as long as $\left.H<H_{c}\right)$.

The energy-gap parameters $P_{i}( \pm)$ shown in Fig. 4 were deduced from a least-squares fitting of the experimental results to the Eqs. (13), (15), and (28). The $\sigma$ dependences of $P_{i}( \pm)$, which were obtained in a self-consistent manner, show no deviations from the simple power-law behavior assumed by Eq. (27). The parameters so deduced, together with their uncertainties, are given in Table I, and it is apparent that $P_{6}(+)$ differs substantially from $\frac{1}{6} g_{\text {eff }} \mu_{B} H_{c}$, implying that $\Delta M$ is different from zero. The necessity for including $\Delta M$ may be seen directly in the measurements

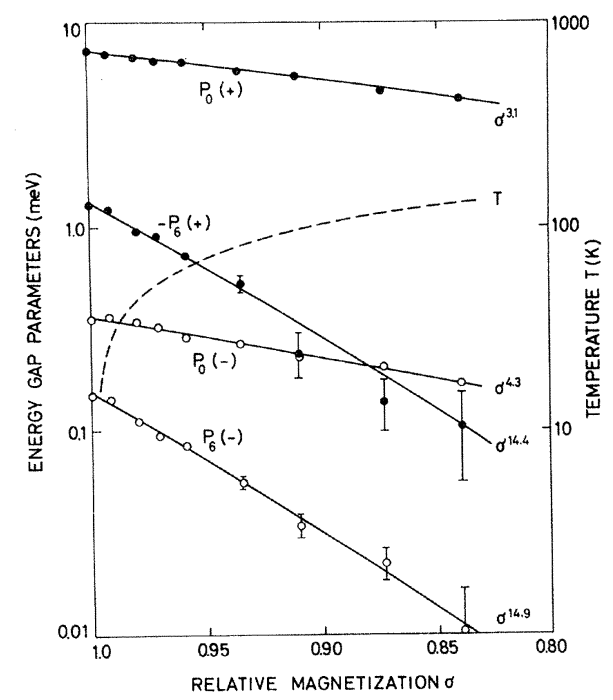

FIG. 4. Anisotropy parameters $P_{i}( \pm)$ in $\mathrm{Tb}$ as a function of magnetization, deduced from the results of Fig. 3 and similar measurements at different temperatures. 
of Fig. 3, since it is responsible for the difference between the slopes of the straight lines obtained for fields applied in the easy and hard directions. The appropriate $g$ value for the macroscopic hexagonal anisotropy is the effective value defined by Eq. (30) of I as used in Table I, $g_{\text {eff }} \mu_{B} H_{c}$.

\section{COMPARISON WITH STATIC EXPERIMENTS}

Deviations from the simple spin-wave renormalization theory of Cooper ${ }^{3}$ or the presence of two-ion $\lambda$ terms, which are not of magnetoelastic origin, may be detected by a comparison of the energy-gap parameters $P_{i}( \pm)$ with the corresponding macroscopic parameters deduced from static measurements ${ }^{22}$ as given by Eq. (22). The temperature dependence of a parameter deduced from static experiments is usually fitted to the temperature law predicted by Callen and Callen ${ }^{2}$ for the (single-ion) term of lowest rank which contributes to the parameter in question. The fits which are obtained based on these assumptions may be quite good, as is the case, e.g., for $C$ and $A$ in $\mathrm{Tb},{ }^{9}$ but deviations occur, especially in the low-temperature region which we are considering $(\sigma>0.8)$. At low temperatures the terms of higher rank may contribute appreciably to the $\sigma$ dependence, and the applicability of the CallenCallen theory in the case of a highly anisotropic system is questionable. Because of these limitations we have fitted a simple power-law $\sigma$ dependence to the experimental results obtained for the magnetostriction parameters $C, A,{ }^{9}$ and $H_{0},{ }^{26,27}$ in the temperature interval corresponding to $\sigma>0.8$. The results are given in Table $\Pi$. The standard deviations, which are also given, are deduced from the statistical scatter of the experimental results and do not include systema-

TABLE I. Anisotropy parameters $P_{i}( \pm)$ and $g_{\text {eff }} \mu_{B} H_{c}$ for $\mathrm{Tb}$ derived from the energy-gap measurements as described in the text. The zero-temperature values of the parameters are given in $\mathrm{meV}$, whereas the magnetization exponents, which describe the temperature dependence of the parameters, are pure numbers. Parameters independent of the actual shape of the crystal may be obtained by subtracting the dipolar contributions $N_{c} g \mu_{B} M$ and $N_{\perp} g \mu_{B} M$ from $P_{0}(+)$ and $P_{0}(-)$, respectively.

\begin{tabular}{crr}
\hline \hline \multicolumn{2}{c}{ Anisotropy parameter } & \multicolumn{1}{c}{ Exponent } \\
\hline$P_{0}(+)$ & $7.22 \pm 0.07$ & $3.07 \pm 0.1$ \\
$P_{6}(+)$ & $-1.34 \pm 0.04$ & $14.4 \pm 0.9$ \\
$P_{0}(-)$ & $0.361 \pm 0.008$ & $4.3 \pm 0.3$ \\
$P_{6}(-)$ & $0.154 \pm 0.003$ & $14.9 \pm 0.8$ \\
$g_{\text {eff }} \mu_{B} H_{c}$ & $0.290 \pm 0.005$ & $15.0 \pm 0.6$ \\
$N_{c} g \mu_{B} M$ & 0.180 & 1 \\
$N_{\perp} g \mu_{B} M$ & 0.058 & 1 \\
\hline \hline
\end{tabular}

tic experimental errors. The elastic constants for $\mathrm{Tb}$ have been measured by Palmer et $a l_{.}{ }^{28}$ as function of temperature and in a field of $25 \mathrm{kOe}$ applied along an easy axis, by which domain effects are eliminated. $c_{66}$ and hence $c_{\gamma}$ is strongly influenced by magnon-phonon interactions, ${ }^{12,13}$ and we use a value which is $(7 \pm 4) \%$ greater than the one obtained from experiment. From the magnetostriction coefficients and the elastic constants, the magnetoelastic coupling parameters, $\sigma c_{\gamma} C$, etc., may be deduced, and they are also given in Table II. Notice that we have included implicitly a factor $1 / \sigma$ in the definitions of $c_{\gamma}$ and $c_{\epsilon}$ following from the factor $1 / J$ in Eq. (5). We remark that if a system is magnetoelastically isotropic $\left[\mathfrak{F}_{\mathrm{cf}-\mathrm{e}},(6)\right.$, independent of a coordinate transformation] then the parameters in (6) are related. Among these relations are $A=0$ and $\sigma c_{\epsilon} H_{0}=4 \sigma c_{\gamma} C$, and it is interesting to notice that the last relation is fulfilled almost exactly in $\mathrm{Tb}$. If terms of higher rank than $l=2$ for $C$ and $H_{0}$ and $l=4$ for $A$ in the Eqs. (10) and (24) and twoion magnetoelastic contributions are neglected then the Callen-Callen theory predicts a renormalization of $\sigma c_{\gamma} C$ and $\sigma c_{\epsilon} H_{0}$ proportional to $\sigma^{3}$ and of $\sigma c_{\gamma} A$ proportional to $\sigma^{10}$. The low-temperature theory of Lindgard and Danielsen ${ }^{23}$ predicts somewhat higher exponents, 3.4 and 14.6 for $\sigma c_{\gamma} C$ and $\sigma c_{\gamma} A$, respectively. The experimental exponents given in Table II, which are smaller than those suggested by the theories, may indicate the importance of two-ion contributions.

The magnetostriction coefficient $H_{0}$ is derived partly from the experiments of DeSavage and Clark $^{26}$ and Du Plessis, ${ }^{27}$ which were performed in the paramagnetic phase, and partly from the amplitude of the magnon-phonon interaction at low temperatures as described in II. $C$ and $\boldsymbol{A}$ and the $\alpha$ strains were measured by Rhyne and Legvold. ${ }^{9} B_{66}^{(i)}$ in Eq. (6) and the corresponding

TABLE II. $\gamma$ - and $\epsilon$-magnetostriction parameters of Tb. $C$ and $A$ are obtained from Ref. 9 and $H_{0}$ is deduced in II. The magnetoelastic coupling parameters, $\sigma c_{\gamma} C$ etc., given in units of meV, are obtained by using the measured elastic constants (from Ref. 27), as described in the text. The exponents give the $\sigma$ dependence of the parameters for $\sigma>0.8$.

\begin{tabular}{|c|c|}
\hline Magnetostriction parameter & Exponent \\
\hline$C \times 10^{3} \quad 4.18 \pm 0.02$ & $2.42 \pm 0.04$ \\
\hline$A \times 10^{3} \quad 2.11 \pm 0.02$ & $7.5 \pm 0.15$ \\
\hline$H_{0} \times 10^{3} \quad 18.5 \pm 2$ & $1.76 \pm 0.04$ \\
\hline $15.0 \pm 0.6$ & $2.65 \pm 0.04$ \\
\hline $7.6 \pm 0.3$ & $7.7 \pm 0.16$ \\
\hline$\sigma c{ }_{\epsilon} H_{0} \quad 62 \quad \pm 7$ & $1.99 \pm 0.04$ \\
\hline
\end{tabular}


two-ion terms introduce a $\phi$ dependence of the $\alpha$ strains. In the interpretation of their results, Rhyne and Legvold neglected this possibility; these terms would then appear as a difference between $C$ deduced from $b$-axis data and from $a$-axis data, whereas $A$ would be unaffected. In their experiment Rhyne and Legvold ${ }^{9}$ detected such a difference for $C$, which we interpret as arising from a $\phi$ dependence of $\epsilon^{\alpha, i}$, with a magnitude of about $0.15 \times 10^{-3} \times \sigma^{7.3}$. The appropriate $C$ value is the mean of the $a$-and $b$-axis data which is the one given in Table II. The neglect of a $\phi$-dependent $\alpha$ strain of this magnitude will appear in $\delta_{0}(-)$ in our results with a contribution equal to $-3 \times 10^{-4} \mathrm{meV}$, which is entirely negligible. Bartholin et $a l .{ }^{29}$ have isolated the contributions to the $\alpha$ strains from the (isotropic) exchange interaction by studying the change of the Néel temperature with uniaxial pressure. Combining these measurements with the $\alpha$-strain measurements, ${ }^{9}$ we deduce an $\alpha$-strain contribution to $P_{0}(+)$ equal to $-0.43 \times \sigma^{1.4} \mathrm{meV}$.

The magnetic anisotropy parameters $K_{l}^{m}$, defined by Eq. (19), have been measured by torque and magnetization experiments. Rhyne and Clark $^{30}$ have determined $K_{2}$ in the easy direction by torque measurements and deduced $K_{6}^{6}$ from the magnetostriction measurements. ${ }^{9}$ Feron et al..$^{31}$ have obtained $K_{2}, K_{4}$, and $K_{6}^{6}$ by magnetization measurements, where $K_{2}$ and $K_{4}$ are the effective anisotropy parameters in the easy direction. $K_{6}^{6}$ determined in these experiments should be directly comparable with the critical field $H_{c}$ obtained in the present experiment through the relation (23). As shown in Fig. 5, the critical fields deduced by the different techniques agree

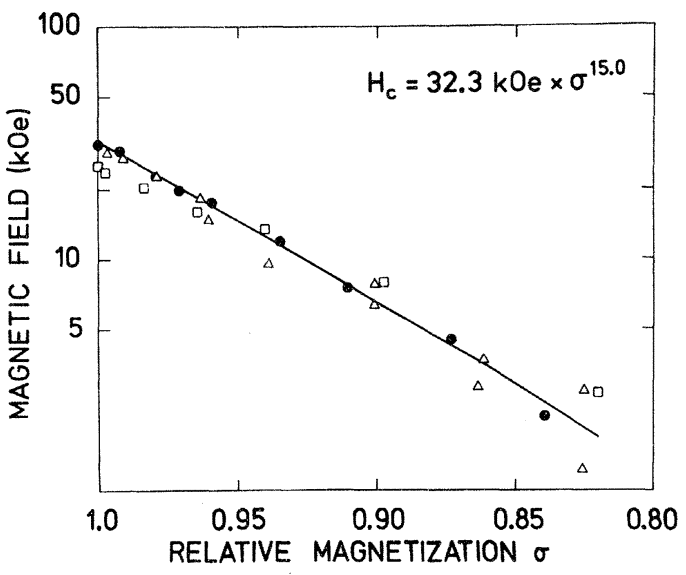

FIG. 5. Critical field $H_{c}$ as function of magnetization. The closed circles are the results obtained by the present experiments on $\mathrm{Tb}$. The open symbols are the results of the measurements by Rhyne and Clark ( $\Delta$, Ref. 30$)$ and by Feron et al. ( $\square$, Ref. 31). satisfactorily. From the experimental result for the magnetic anisotropy parameter $\mathrm{s}^{30,31}$ and for the $\epsilon$ strain (Table II) we have calculated the macroscopic value for $P_{0}(+)+P_{6}(+)$ according to the Eqs. (21) and (22a). $P_{0}(+)+P_{6}(+)$ is equal to $A(0)+B(0)$ when the magnetization is along an easy axis. The comparison is shown in Fig. 6. The large absolute uncertainties of the the static anisotropy parameters (of the order of $25 \%$ ) may disguise a difference of the order $\delta_{0}(+)+\delta_{6}(+) \approx 0.4 \pm 1.0 \mathrm{meV}$, but the similarities of the $\sigma$ dependence suggest that the difference is probably quite small.

The static expression for $A(0)-B(0)$ involves only the $\gamma$-strain contributions and the critical field as given by Eq. (22b). In Table III we compare the static and dynamic values for $P_{i}(-)$. The agreement between the two values for $P_{0}(-)$ is extremely good, with respect to both the absolute magnitude at zero temperature and the temperature dependence. In the case of $P_{6}(-)$ we obtain a significant difference $\delta_{6}(-)$. To elucidate this difference further we have subtracted the (static) magnetoelastic contribution $10 c_{\gamma} A C$ from both the microscopic and macroscopic results for $P_{6}(-)$ :

$$
\begin{aligned}
& \left(P_{66}\right)_{\text {micr. }}=P_{6}(-)-10 c_{\gamma} A C, \\
& \left(P_{66}\right)_{\text {macr. }}=g_{\text {eff }} \mu_{B} H_{C}-8 c_{\gamma} A C-10 c_{\gamma} A C .
\end{aligned}
$$

The ratio between the dynamic and static hexagonal anisotropies of the unstrained lattice is close to 2 and is practically independent of temperature. Note that this part of the static anisotropy $\left(P_{66}\right)_{\text {macr. }}$ has the opposite sign to the total static basalplane anisotropy $K_{6}^{6}$, and that it renormalizes quite slowly with temperature, as $\sim \sigma^{6}$.

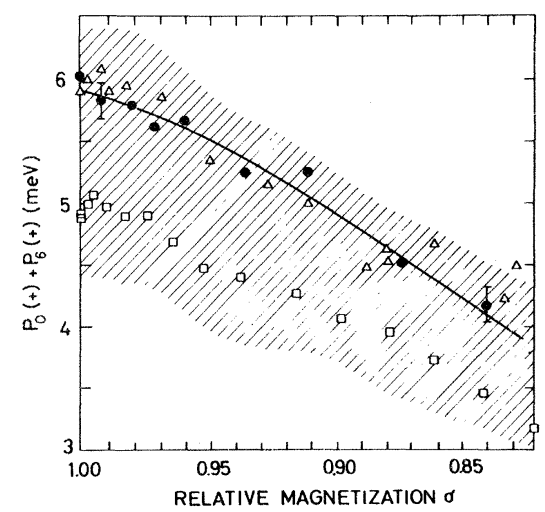

FIG. 6. Comparison of the energy-gap parameter $A(0)+B(0)$ in the easy direction of Tb (closed circles) with the static values deduced from the magnetic anisotropy measurements as described in the text ( $\Delta$ from Ref. 30 and $\square$ from Ref. 31). The cross-hatched area shows the probable region in which the macroscopic parameter lies when combining the two different anisotropy measurements. 
TABLE III. Comparison of static and dynamic energygap parameters. The microscopic (dynamic) anisotropy parameters are from Table I, and the macroscopic parameters are deduced from the parameters given in Tables I and II according to Eq. (22b).

\begin{tabular}{|c|c|c|}
\hline \multicolumn{2}{|c|}{ Anisotropy parameter } & \multirow{3}{*}{$\begin{array}{l}\text { Exponent } \\
4.3 \pm 0.3 \\
4.2 \pm 0.13\end{array}$} \\
\hline & $\{$ dyn. $0.361 \pm 0.008$ & \\
\hline$P_{0}(-)$ & $\begin{cases}\text { sta. } & 0.367 \pm 0.011\end{cases}$ & \\
\hline$\delta_{0}(-)$ & \pm 0.014 & $\cdots$ \\
\hline$P_{f}(-)$ & $\begin{cases}\text { dyn } & 0.154 \pm 0.003\end{cases}$ & $14.9 \pm 0.8$ \\
\hline$P_{6}(-)$ & $\{$ sta. $\sim 0 \quad \pm 0.03$ & $\cdots$ \\
\hline$\delta_{6}(-)$ & $0.126 \pm 0.010$ & $5.9 \pm 0.3$ \\
\hline & (dyn. $-0.167 \pm 0.013$ & $5.6 \pm 0.5$ \\
\hline$P_{66}$ & $\{$ sta. $-0.292 \pm 0.024$ & $5.8 \pm 0.4$ \\
\hline
\end{tabular}

\section{DISCUSSION}

From the measurements of the field dependence of the energy gap at zero wave vector in the spinwave spectrum presented in this paper, some of which have been published earlier, ${ }^{32}$ we deduce a strong axial anisotropy in $\mathrm{Tb}$, which has also been observed by torque ${ }^{30}$ and magnetization ${ }^{31}$ measurements. Further, we find that the axial anisotropy depends sensitively on the orientation of the magnetic moments in the basal plane. The large value for $P_{6}(+)$ which we obtain can hardly be explained within a single-ion model for the anisotropy, Eqs. (17) and (18), as $\Delta M=P_{6}(+)$ $-\frac{1}{6} g_{\text {eff }} \mu_{B} H_{c}=-1.39 \times \sigma^{14.3} \mathrm{meV}$ is an order of magnitude larger than the $\gamma$-strain terms in $P_{i}(-)$. If $C, A$, and $\Delta M$ were all of magnetoelastic singleion origin, then the magnitude of $\Delta M$ would require $C$ and $A$ to be differences between terms which were 10 times larger and with different $\sigma$ dependences. Most of the contributions to $\Delta M$ are presumably of two-ion origin. By the inclusion of two-ion interactions a general expression for the energy gap was deduced in I [Eqs. (21)-(23)]. Two types of two-ion interactions may contribute to $P_{6}(+)$, those for which $l+m$ and $l^{\prime}+m^{\prime}$ are both even, and those for which they are both odd. How ever, when $\frac{1}{6} g_{\text {eff }} \mu_{B} H_{c}$ is subtracted from $P_{6}(+)$, the remaining part $\Delta M$ is mostly composed of twoion contributions for which $l+m$ and $l^{\prime}+m^{\prime}$ are both odd; these are the same interactions which give rise to the $\vec{q}$-dependent anisotropy, $\mathfrak{e}(\overrightarrow{\mathrm{q}})$ derived in $I$. We found in $I$ that $\mathfrak{e}(\vec{q})$ gives rise to an interplanar contribution to $P_{6}(+)$ equal to -1.6 $\mathrm{meV}$ at zero temperature. On the basis of this strong correlation between $\mathfrak{e}(\overrightarrow{\mathrm{q}})$ and $\Delta M$, both with respect to their magnitudes and their $\sigma$ dependences, combined with the arguments above we conclude that $\Delta M$ is of two-ion origin. A possible strain dependence of these couplings $(\lambda \neq 0)$ will not affect the $\gamma$ or $\alpha$ strains or the difference $\delta_{6}(+)$.

In I anisotropic two-ion couplings were found to be important in $\mathrm{Tb}$, giving rise to $\overrightarrow{\mathrm{q}}$-dependent anisotropy, and there is therefore the possibility of large two-ion contributions to the magnetic anisotropy at $q=0$. The evidence for the two-ion origin of $\Delta M$ is convincing. The differentiation between single-ion and two-ion contributions to $P_{0}(+)$ is more uncertain. An attempt may be based on the studies by $\mathrm{H} \phi \mathrm{g}$ and Touborg, ${ }^{33}$ who have determined the crystal-field anisotropy by magnetization measurements on single-crystal alloys of rare-earth metals diluted in $\mathrm{Y}, \mathrm{Lu}$, and $\mathrm{Sc}$, and the equivalent torque measurements on dilute alloys of rare-earth ions in Gd by Chikazumi et $a l .^{34} \mathrm{Asada}^{35}$ has later corrected the Gd results for the effect of finite concentration of the rareearth impurities. The remarkable consistency of the crystal-field parameters obtained for a rareearth ion, when placed in the different systems, supports a use of these parameters as a measure of the single-ion anisotropy present in the pure metal. The results of $\mathrm{H} \phi \mathrm{g}$ and Touborg ${ }^{33}$ and of Asada $^{35}$ both suggest a single-ion contribution to the axial anisotropy $P_{0}(+)$ in $\mathrm{Tb}$ of the order of $2 \mathrm{meV}$, leaving $5 \mathrm{meV}$ as being of two-ion origin. The single-ion magnetoelastic part of $P_{0}(+)$ is probably small, as shown by the estimated $\alpha$ strain contribution which is compensated almost exactly by $c_{\gamma}\left(2 C^{2}+A^{2}\right)$ and the dipolar term. The $\overrightarrow{\mathrm{q}}$-dependent axial anisotropy in $\mathrm{Tb}, \mathcal{K}(\overrightarrow{\mathrm{q}})$ in $\mathrm{I}$, indicates that part of the interplanar contributions to $P_{0}(+)$ is negative. Such negative contributions may be connected to two-ion couplings of low rank which would be consistent with the tendency found by Boutron, ${ }^{36}$ who considered the two-ion couplings for which $l=l^{\prime}=1$. That the total two-ion axial anisotropy at $q=0$ is found to be positive indicates the importance of couplings of higher rank.

The only couplings which appear in $P_{0}(-)$ are those for which $\mu$ (and hence $\lambda$ ) are different from zero, Eq. (23) of I. By expressing $P_{0}(-),(17 \mathrm{c})$, in terms of $C$ and $A$, which are defined by Eq. (9), we have included all contributions of magnetoelastic origin. Polarization and spin-orbit coupling effects on the susceptibility of the conduction electrons may introduce terms for which $\lambda$ is nonzero, when combined with the indirect $s-f$ exchange interaction, $J \mathfrak{g}(0) \approx 8 \mathrm{meV}$ in $\mathrm{Tb}$. From the order-of-magnitude estimate in I [mechanism (v)] we expect contributions to the energy -gap parameters $P_{i}(-)$ of the order of $\left(m+m^{\prime}\right)^{2}$ times $8 \times 10^{-3} \mathrm{meV}$. Two-ion terms arising from this mechanism, for which $\mu$ is different from zero, would appear in $\delta_{0}(-)$ and $\delta_{6}(-)$, Eq. (26). There is no indication of such a contribution to $\delta_{0}(-)$, whereas $\delta_{6}(-)$ is different from zero, as appears 
from Table III. In fact, terms for which $\mu$ is equal to 1 or 2 would account for the factor of 2 between the dynamic and static values of $P_{66}$, and a term like $K_{222}^{222}(0)$ would, according to the simple renormalization theory [Eqs. (32) and (33) of I], be proportional to $\sigma^{8}-\sigma^{12}$, which is fairly close to the observed $\sigma^{6}$ dependence. A two-ion contribution to $P_{6}(-)$ of this order of magnitude is consistent with the estimated interplanar term arising from the $\overrightarrow{\mathrm{q}}$-dependent anisotropy, $D(\overrightarrow{\mathrm{q}})$ in I $\left[\approx P_{6}(-)\right]$.

The magnon-magnon interaction ${ }^{23,24}$ may introduce zero-point deviations between static and dynamic variables. In the theory of Lindgård and Danielsen ${ }^{23}$ two-ion anisotropy is neglected, and they predict a detectable deviation only in the case of the sixfold basal-plane anisotropy $V_{66}$, for which the dynamic value should be about half the static one. This is in accordance with the observed behavior of $P_{66}$ in $\mathrm{Tb}$, but introduces in Dy an effect which is opposite in sign to the one deduced from experiments. ${ }^{37}$ Further, the $\sigma$-dependence of $P_{66}$ expected from the theory of Lindgård and Danielsen is inconsistent with the experimental results. The low-temperature theory predicts an exponent for the dynamic $V_{66}$ which is about twice the value of 20 given by the simple $\frac{1}{2} l(l+1)-1$ law. The small exponent observed for $P_{66}$ allows us to draw the conclusion that the most important contributions to $P_{66}$ are of two-ion origin. A two-ion origin of $P_{66}$ or the small $o$ exponent obtained further indicates that the zeropoint difference between the dynamic and the static $P_{66}\left(\lambda+l+l^{\prime}=6\right)$ should be considerably smaller than the one deduced ${ }^{23}$ for $V_{66}(l=6)$. This leads us to propose mechanism ( $v$ ) in I for the observed difference $\delta_{6}(-)$ as a more probable explanation than the magnon-magnon interaction.

The possibility of several types of contributions to the deduced parameters does not allow any definite conclusions about deviations from the Callen-Callen theory, ${ }^{2}$ generalized to the dynamic case by Cooper, ${ }^{3}$ which is predicted ${ }^{23,24}$ for a strongly anisotropic basal-plane ferromagnet. For this purpose Dy may serve as a better candidate than $\mathrm{Tb}$, because of the presence of a large basalplane anisotropy in Dy. Nicklow and Wakabayashi ${ }^{37}$ have observed an energy gap in Dy equal to 3.2 $\mathrm{meV}$ at zero temperature by neutron scattering, whereas the value obtained from the static measurements $^{22,31}$ is $2.6 \pm 0.4 \mathrm{meV}$. The large dif ference suggested by these numbers deserves further study. By similar measurements of the field dependence of the energy gap in Dy a distinction between the $\delta_{i}( \pm)$ may be obtained, together with a more reliable determination of the critical field ( $135 \mathrm{kOe}$ at zero temperature). A $\phi$ de- pendence of the susceptibility of the conduction electrons which may explain the peculiar behavior of $P_{66}$ in $\mathrm{Tb}$ may not account for the large differ-. ence of the order of $0.6 \mathrm{meV}$ in Dy. The softening of the velocity of transverse sound waves propagating in the basal plane at fields close to the critical field is presumably not affected by the magnon-magnon interaction (only dynamic parameters are involved), whereas the presence of strain-independent $\lambda$ terms will influence the critical behavior. Ultrasonic studies of the transition at the critical field in Tb and Dy could clarify the origin of the discrepancies between the static and the dynamic values for the energy gaps. See Note added in proof.

\section{SUMMARY}

In the three papers I, II, and III we have presented a detailed experimental and theoretical analysis of the magnetic excitations propagating in the $c$ direction of $\mathrm{Tb}$. The ground state of the ionic moments in $\mathrm{Tb},{ }^{25,38}$ which are ferromagnetically ordered at low temperatures, is presumably close being a pure $J_{z}=J=6$ state; so the spinwave approach is applicable. The description of the spin waves propagating in the $c$ direction of a hexagonal solid is especially simple because the effective Hamiltonian is close to being invariant with respect to a rotation of the magnetization vector around the $c$ axis by an angle which is a multiple of $\frac{1}{3} \pi$. If the $c$ axis is considered to be an effective sixfold axis, then the absence of acoustic-optical couplings implies that the doublezone representation for the hcp lattice is valid. The direct mixing of spin space variables accomplished by the spin-orbit coupling of the conduction electrons causes a lowering of the effective symmetry. The spin-orbit coupling introduces a further complication, as it gives rise to a deviation between the direction of magnetization and the direction in which the conduction electrons are polarized. This deviation may introduce couplings violating the selection rules for a simple ferromagnet, as is the case for the acousticoptical magnon-phonon interaction observed in $\mathrm{Tb}$.

A general dispersion relation for spin waves propagating in the $c$ direction of a basal-plane ferromagnet was derived. The different forces acting on the ionic magnetic moments may be divided into the three classes: single-ion, twoion, and polarization-dependent two-ion spin interactions. Three- or many-ion interactions can be decoupled into effective two-ion interactions. The positional dependence of all three types of coupling appears as static magnetostrictive contributions to the magnon energies and as a dynamic inter- 
action between the magnons and the lattice vibrations.

Different aspects of the magnetic anisotropy in $\mathrm{Tb}$ were studied by measuring the change of the magnon dispersion relation when an external magnetic field was applied along a symmetry direction in the basal plane. By such an external perturbation of the magnon spectrum it is possible to determine the two Bogoliubov energy components $A(\overrightarrow{\mathrm{q}})+B(\overrightarrow{\mathrm{q}})$ and $A(\overrightarrow{\mathrm{q}})-B(\overrightarrow{\mathrm{q}})$. If $B(\overrightarrow{\mathrm{q}})$ is nonzero, then the total magnetization, which is the sum of the $z$ components of the ionic moments, does not commute with the spin-wave Hamiltonian. $B(\overrightarrow{\mathrm{q}})$ vanishes identically for a $c$-axis magnet, whereas deviations from the fully aligned ground state assumed in a spin-wave approximation may be important in a basal-plane magnet..$^{23,24}$ For a $c$ axis magnet, only terms for which $\mu=0$ and $m=-m^{\prime}=1$ in Eq. (7) of I can contribute to the dispersion of the $c$-axis magnons, and $A(\overrightarrow{\mathrm{q}})+B(\overrightarrow{\mathrm{q}})$ and $A(\overrightarrow{\mathrm{q}})-B(\overrightarrow{\mathrm{q}})$ are degenerate $[B(\overrightarrow{\mathrm{q}})=0]$. The information about magnetic anisotropy which may be obtained from the dispersion of the magnons in this spin configuration is thus rather limited. The basal-plane spin configuration which occurs in $\mathrm{Tb}$ and Dy is more informative, and the possibility of changing the orientation of the moments within the basal plane of $\mathrm{Tb}$ has led to the observation of spin couplings of high rank. $\lambda+l+l^{\prime} \geqslant 6$ for the terms which contribute to the $\phi$-dependent anisotropy, $P_{6}( \pm)$, and $\mathfrak{C}(\overrightarrow{\mathrm{q}})$ and $\mathfrak{D}(\overrightarrow{\mathrm{q}})$ derived in I.

In II we isolated the effects of the direct coupling between magnons and transverse phonons at finite wave vector. With in certain limitations, we determined explicitly the terms in the energy gap at zero wave vector arising from magnetoelastic coupling ( $\Delta M$ may include magnetostrictive twoion contributions). By this procedure we accounted for most of the effects of coupling between the spin system and the lattice. Magnetostrictive distortions of the lattice may enhance existing two-ion anisotropy, and this effect may in principle be investigated by applying a uniaxial pressure.

By the determination of $A(\overrightarrow{\mathrm{q}}) \pm B(\overrightarrow{\mathrm{q}})$ at both zero and finite wave vector we have separated the constant and the $\vec{q}$-dependent anisotropy. The results indicate that all three types of spin couplings contribute to the constant anisotropy. The single and two-ion contributions seem to be of comparable importance, and the comparison between the static and dynamic anisotropy suggests the presence of a small polarization-dependent term. The $\vec{q}$ dependence of the unperturbed energy parameters $A(\overrightarrow{\mathrm{q}}) \pm B(\overrightarrow{\mathrm{q}})$ reflects intrinsic properties of the spin system and is due to two- (and many-) ion couplings. The different kinds of spin interactions present in $\mathrm{Tb}$ can be listed according to their relative importance on the magnon energies at zero temperature as follows: (i) Heisenberg interaction, $\sim 7 \mathrm{meV}$; (ii) two-ion anisotropy, $\sim 3 \mathrm{meV}$; (iii) single-ion anisotropy, $\sim 3 \mathrm{meV}$; (iv) magnetoelastic coupling, $\sim 0.5 \mathrm{meV}$; (v) polarization-dependent two-ion anisotropy, $\sim 0.2$ meV. The determination of the different contributions to the energy suffers from considerable uncertainty which is reflected in the typical energies listed above.

The behavior of the spin system in $\mathrm{Tb}$ is dominated by two-ion couplings between the ionic moments, (i) and (ii). In the heavy-rare-earth metals the electric multipole interactions [mechanism (vi) in I, $l$ and $l^{\prime} \neq 0$ ] are of minor importance in comparison with the indirect exchange interaction via the conduction electrons. The twoion anisotropy which has been observed to be present in $\mathrm{Tb}, \mathrm{Dy}$, and $\mathrm{Er}$ is presumably due to the influence of mechanisms (i) and (ii), described in $I$, on the exchange interaction. A theoretical calculation of the indirect exchange interaction from first principles would be extremely complicated, as it requires a knowledge of the relativistic wave function of both the localized $4 f$ electrons and the conduction electrons and a correct treatment of screening. Relativistic effects such as the spin-orbit coupling of the conduction electrons, (ii), may turn out to be as important as the Kaplan-Lyons terms, (i).

Utilizing the systematic behavior through the heavy-rare-earth series, the microscopic origin of magnetic anisotropy in these metals may be further elucidated experimentally. The behavior of the elementary excitations of a magnetically ordered metal is determined by certain linear combinations of general spin couplings which depend on the orientation of the ordered moments. When comparing the magnetic properties of the rare-earth metals the terms appearing in the linear combinations, $A(\overrightarrow{\mathrm{q}}) \pm B(\overrightarrow{\mathrm{q}})$, in identical spin configurations may scale differently according to the rank $l$ and to the origin of the terms. A rough estimate of the scaling of the anisotropy energy $E_{A}$ in the heavy-rare-earth metals due to orbital modifications $(\propto L)$ of the exchange interaction $(\propto S)$ is $E_{A} \propto L S=(2-g)(g-1) J^{2}$. This expression should not be taken too literally, as, e.g., the spin-orbit coupling, (ii), may give rise to two-ion anisotropy in $\mathrm{Gd}(g=2)$, as mentioned in I.

\section{ACKNOWLEDGMENTS}

The authors of the preceding three papers are grateful to A. R. Mackintosh, who participated in the early stages of the work, for valuable suggestions, stimulating interest, and a critical 
reading of the manuscripts. Helpful discussions with R. J. Birgeneau, R. J. Elliott, P. A. Lindgård, O. Danielsen, P. Bak, and M. Nielsen are also gratefully acknowledged.

Note added in proof. The polarization-dependent two-ion coupling is most likely interpreted as a dynamic effect which vanishes in the limit of zero energy. The behavior of the long-wavelength phonons ( $\approx$ zero energy) should be adequately described by the macroscopic anisotropy parameters [see a discussion by Jensen (to be published)].
${ }^{1}$ W. P. Mason, Phys. Rev. 96, 302 (1954).

${ }^{2}$ E. Callen and H. B. Callen, Phys. Rev. 139, A455 (1965).

${ }^{3}$ B. R. Cooper, Phys. Rev. 169, 281 (1968); in Solid State Physics, edited by F. Seitz and D. Turnbull (Academic, New York, 1968), Vol. 21, p. 393.

${ }^{4}$ D. A. Goodings and B. W. Southern, Can. J. Phys. 49 , 1137 (1971).

${ }^{5}$ E. A. Turov and V. G. Sharov, Fiz. Tverd. Tela 7, 217 (1965) [Sov. Phys.-Solid State 7, 166 (1965)].

${ }^{6}$ W. E. Evenson and S. H. Liu, Phys. Rev. 178, 783 (1969).

${ }^{7}$ M. Rosen and H. Klimker, Phys. Rev. B 1 , 3748 (1970)。

${ }^{8}$ B. R. Cooper, Phys. Rev. Lett. 19, 900 (1967).

${ }^{9}$ J. J. Rhyne and S. Legvold, Phys. Rev. 138, A507 (1965).

${ }^{10}$ P. A. Lindgård, J. Phys. (Paris) 32, C1-238 (1971).

${ }^{11}$ F. Keffer, Handbuch Der Physik, edited by S. Flügge (Springer, Berlin, 1966), Vol. XVIII/2, p。 168 .

${ }^{12}$ J. Jensen, Int. J. Magn. 1, 271 (1971).

${ }^{13}$ J. Jensen, Ris $\varnothing$ report No. 252, 1971 (unpublished).

${ }^{14}$ H. Chow and F. Keffer, Phys. Rev. B 7, 2028 (1973).

${ }^{15}$ S. H. Liu, Int. J. Magn. 3,327 (1972)。

${ }^{16} \mathrm{D}$. T. Vigren and S. H. Liu, Phys. Rev. Lett. 27, 674 (1971); L. W. Hart and J. L. Stanford, ibid. 27, 676 (1971).

${ }^{17}$ D. T. Vigren and S. H. Liu, Phys. Rev. B $\underline{5}, 2719$ (1972).

${ }^{18}$ M. Nielsen, H. Bjerrum Møller, P. A. Lindgård, and A. R. Mackintosh, Phys. Rev. Lett. 25, 1451 (1970).

${ }^{19}$ R. J. Elliott, A. P. Young, and S. R. P. Smith, J. Phys. C 4, L317 (1971); J. R. Sandercock, S. B. Palmer, R. J. Elliott, W. Hayes, S. R. P. Smith, and A. P. Young, J. Phys. C 5, 3126 (1972).

${ }^{20}$ J. K. Kjems, G. Shirane, R。 J. Birgeneau, and L. G。 Van Uitert, Phys。 Rev. Lett. 31, 1300 (1973)。
${ }^{21}$ S. B. Palmer (private communication).

${ }^{22}$ J. J. Rhyne, Magnetic Properties of Rare Earth Metals, edited by R. J. Elliott (Plenum, London, 1972), Chap. 4.

${ }^{23}$ P. A. Lindgård and O. Danielsen, Phys. Rev. B 11, 351 (1975); O. Danielsen, Ris $\varnothing$ report No. 295, 1973 (unpublished).

${ }^{24}$ T. Egami, J. Phys. C $\underline{5}$, L85 (1972); M.S. S. Brooks and T. Egami, J. Phys. C $\underline{6}, 513$ (1973).

${ }^{25}$ D. E. Hegland, S. Legvold, and F. H. Spedding, Phys。 Rev. 131, 158 (1963).

${ }^{26} \mathrm{~B} . \mathrm{F}$. DeSavage and A.E. Clark, in Proceedings of the Fifth Rare Earth Research Conference, Ames, Iowa, 1965 (unpublished).

${ }^{27}$ P. De V. Du Plessis, Philos. Mag. 18, 145 (1968).

${ }^{28}$ S. B. Palmer, E. W. Lee, and M. N. Islam, Proc. R. Soc. A 338,341 (1974).

${ }^{29} \mathrm{H}$. Bartholin, J. Beille, D. Bloch, P. Boutron, and J. L. Feron, J. Appl. Phys. 42, 1679 (1971).

${ }^{30}$ J. J. Rhyne and A. E. Clark, J. Appl。Phys。 38, 1379 (1967).

${ }^{31}$ J. L. Feron, G. Huy, and R. Pauthenet, CRNS report No. 180, Paris, 1970, p. 19 (unpublished).

${ }^{32}$ H. Bjerrum Møller, J. C. G。 Houmann, J. Jensen, P. A. Lindgård, M. Nielsen, and A. R. Mackintosh, Ris $\varnothing$ report No. 256, 1972, p. 223 (unpublished).

${ }^{33} \mathrm{~J}$. Hog and P. Touborg, Phys. Rev. B 11, 520 (1975).

${ }^{34}$ S. Chikazumi, K. Tajima, and K. Toyama, J. Phys. (Paris) 32, C1-179 (1971).

${ }^{35}$ T. Asada, J. Phys. Soc. Jpn. 35, 85 (1973).

${ }^{36}$ P. Boutron, Phys. Rev. B 9,2971 (1974).

${ }^{37}$ R. M. Nicklow and N. Wakabayashi, Neutron Inelastic Scattering (IAEA, Vienna, 1972), p. 611.

${ }^{38} \mathrm{~A}$. R. Mackintosh and H. Bjerrum Møller, Magnetic Properties of Rare Earth Metals, edited by R. J. Elliott (Plenum, London, 1972), Chap. 5. 